\title{
Plataforma de recursos educativos abiertos para la formación de capital humano en pymes
}

\section{Platform of open educational resources for the training of human capital in SMEs}

\author{
María Dolores Martínez Guzmán ${ }^{1}$ \\ https://orcid.org/ 0000-0003-4523-4655 \\ Dámaris Chávez Maza² \\ http://orcid.org/ \\ Marcela Rojas Ortega ${ }^{3}$ \\ https://orcid.org/0000-0003-1246-5051 \\ Instituto Politécnico Nacional, México
}

Vol. 6(1). Enero-abril. Hamut’ay 2019. Lima-Perú

Recibido: 10-01-2019

Aceptado: 13-04-2019

\section{Cita Recomendada}

Martinez, M., Chávez, D. \& Rojas, M. (2019) Plataforma de recursos educativos abiertos para la formación de capital humano en pymes. Hamut'ay, 6(1), 71-81.

http://dx.doi.org/10.21503/hamu.v6i1.1575

\section{RESUMEN}

Los cambios que se han ido dando a nivel global en la formación del capital humano en el ámbito laboral ha conllevado a que las empresas utilicen estrategias innovadoras que implican una apropiación de las tecnologías en su recurso humano. Por lo que en este estudio se describe la implementación de los Recursos Educativos Abiertos en una plataforma diseñada para la formación de capital humano de las pymes de la ciudad de México, siendo estas las variables de estudio. Se muestra los resultados del Recurso Educativo Abierto desarrollado en una plataforma tecnológica, en su primera fase.

Se tuvo como objetivo describir la fase diagnóstica de la plataforma, la cual ha permitido realizar las tres etapas posteriores de la misma relacionada: a. Diseño, b. Aplicación y c. Validación de la plataforma REA. El estudio fue de tipo exploratorio, ya que se quería conocer cuál era el interés de los usuarios en el uso de este tipo de recurso y descriptivo al permitir detallar el procedimiento que se realizó antes del desarrollo de la plataforma, utilizando para ello un método cuantitativo de recopilación de datos. La muestra estuvo compuesta por 97 empresarios del sector servicios de la Delegación Tlalpan de la Ciudad de México. Los resultados permitieron obtener información relevante para entender la problemática que expresaron los empresarios, de acuerdo a sus respuestas en la encuesta, y la necesidad de utilizar como herramienta los Recursos Abiertos de la Plataforma Tecnológica REA, para formación de capital humano en temas relacionados con la permanencia de su empresa en el mercado.

1 Docente-investigadora del Instituto Politécnico Nacional (IPN), ESCA Tepepan, México. En la línea de investigación Gestión de Conocimiento y Tecnologías de la Información y comunicación en las organizaciones. E-mail: lolita.martinezg@gmail.com

2 Docente-investigadora del Instituto Politécnico Nacional (IPN), ESCA Tepepan, México. En la línea de investigación de Tecnologías de la Información y comunicación para la formación de recursos humanos. E-mail: damaris.mkt.ipn@gmail.com

3 Docente-investigadora del Instituto Politécnico Nacional (IPN), ESCA Tepepan, México. En la línea de investigación educativa en las carreras de negocios. E-mail: mrojaso7@hotmail.com 
Palabras Clave: Recurso Educativo Abierto, tecnologías, capital humano, formación empresarial, pymes.

\begin{abstract}
The changes that have taken place globally in the training of human capital in the workplace have led companies to use innovative strategies that imply an appropriation of technologies in their human resources. So this study describes the implementation of Open Educational Resources in a platform designed for human capital training of SMEs in Mexico City, being these the study variables. It shows the results of the Open Educational Resources developed in a technological platform, in its first phase.

The aim was to describe the diagnostic phase of the platform, which has enabled its three subsequent stages in relation to: a. Design, b. Application and c. Validation of the REA platform. This was an exploratory study since it aimed to know what was the interest of the users in the use of this type of resources; it was also descriptive, since it allowed to detail the procedure that was carried out before the development of the platform, using a quantitative method of data collection. The sample consisted of 97 entrepreneurs from the services sector of the Tlalpan Delegation of Mexico City. The results allowed to obtain relevant information to understand the problems expressed by the entrepreneurs, according to their responses in the survey; and the need to use the Open Resources of the REA Technology Platform as a tool, to train human capital in topics related to the permanence of their company in the market.
\end{abstract}

Keywords: Open educational resource, technologies, human capital, business training, SMEs.

\section{INTRODUCCIÓN}

La formación de capital humano ha sido una constante en el ámbito empresarial, ya que tener personal reclutado con un alto nivel académico, no solo beneficia al trabajador sino también al sector empresarial, por estar estos capacitados con los últimos avances de la ciencia y las tecnologías. Pero la realidad nos lleva a reflexionar, porque en pleno siglo XXI continuamos con déficit de capital humano formado de manera idónea para afrontar estos retos de este siglo, es una problemática que afecta a la industria y al mundo en general, (Román, 2013, Cámara de Comercio, 2017).

Dentro de este contexto las Pequeñas y Medianas Empresas (pymes) no están exentas, al constituir el sector más dinámico de las economías, incentivan la competencia, la generación de ideas, productos y servicios, en América Latina, consti- tuyen entre $95 \%$ al $99 \%$ de sus economías (C.E. INEGI, 2015, p. 15), las cuales están conformadas por capital humano. Sumado a esto, los escenarios que enfrentan las organizaciones se encuentran llenos de incertidumbre, debido, por un lado, a la competencia directa de las grandes organizaciones y por otro, a la falta de formación del capital humano en aspectos de cultura tecnológica, entre otros aspectos relacionados con la gestión de la organización.

En la Ciudad de México, la problemática de formación de capital humano, es un tema muy complejo y requiere un profundo análisis, debido a que estamos viviendo en una economía llena de incertidumbre donde el conocimiento es la base para realizar los negocios con creatividad e innovación, los conocimientos deben adquirirse más rápido, antes que la competencia para lograr la mayor per- 
manencia en el mercado globalizado. De lo cual no están exentas las pymes en México, ya que estas poseen una limitada cultura en tecnologías, no poseen la cantidad suficiente de personal calificado para gestionar las tecnologías de la información y comunicación (TIC), (Diaz, 2017).

En este orden de ideas, Cueto, 2013; De Marco, 2013, mencionan que para ir incrementando los niveles de productividad del país se requiere incentivar la formación del capital humano con el uso adecuado de las TIC, ya que esta ha generado cambios en los procedimientos de trabajo. Para Mejía (2017) la aplicación de las TIC en el capital humano es uno de los factores que inciden en el crecimiento económico, ya que las tecnologías impactan en todas las actividades humanas, empresariales y en la sociedad en general. Asimismo, el mundo empresarial donde están inmersas las pymes se apoya de las TIC para efectivizar sus procesos productivos, abaratar costos de producción, ampliar su mercado a través de redes sociales para tener un mayor número clientes y proveedores, (Gálvez, Riascos \& Contreras, 2014). Desde esta perspectiva se consideró necesario desarrollar recursos digitales, con la finalidad de impartir contenidos en temáticas que apoyaran a la formación de los empresarios de las pymes de la Delegación Tlalpan; debido a las dificultades de espacio y tiempo que tienen para realizar una actualización continua de manera presencial como lo exige el mercado laboral.

Siendo el objetivo de este estudio el describir los resultados del diagnóstico de necesidades de formación y el uso de herramientas tecnológicas para desarrollar contenidos digitales a través del diseño y desarrollo de la interfaz que integra los Recursos Educativos Abiertos en la plataforma tecnológica REA como soporte en la formación de empresarios de las pymes de la delegación Tlalpan de la Ciudad de México.

Delimitando como nuestras variables Recursos Educativos Abiertos en Plataformas Tecnológicas (Variable independiente), la cual es descrita de manera general, ya que el estudio se centra en presentar la fase diagnóstica, cuyos resultados han servido para el diseño y desarrollo de la plataforma REA. Y formación en capital humano (Variable depen- diente), es en la que se quiere incidir si la Plataforma tiene una influencia en mejorar el perfil de los empresarios de la pyme, lo cual será presentado posteriormente en otra publicación, porque esta fase se encuentra en aplicación.

\section{Recursos Educativos Abiertos en Plataformas} Tecnológicas

La incursión de la TIC en el ámbito empresarial que incluyen desde recursos informáticos hasta plataformas de asistencia y capacitación han ido en aumento, siendo utilizadas por las pymes, pues puede apoyar al aprendizaje, (Hirsch, Almaraz \& Ríos, 2015). Al utilizar las herramientas tecnológicas vinculadas con Recursos Educativos Abiertos (REA) contribuyen a mejorar el proceso de formación, debido a que los REA forman parte del aprendizaje soportados por TIC, ya que brindan la facilidad de ser ensamblados, cambiados y/o adaptados, así como ser reutilizados, embebidos o referenciados en otras plataformas y de acuerdo a las características del usuario, es de acceso público y con una amplitud de contenidos digitales en los que pueden estar inmersos texto, audio, imágenes, juegos educativos, MOOC y una diversidad de materiales, (Reyes, Juárez, Martínez \& Sánchez, 2017; Guzmán \& Motz, 2013)

Tomando como base el contexto actual descrito en el que se desarrollan las pymes en la Ciudad de México, el enfoque teórico en el que se sustenta el desarrollo de la Plataforma REA, se basa en las teorías constructivistas sobre el aprendizaje, a través de dicho enfoque, se fundamenta el diseño de contenidos digitales, así como la selección de herramientas digitales para el diseño instruccional de estos recursos virtuales para impartir cursos de capacitación en el lugar de trabajo para formar al capital humano. La arquitectura e interfaz de la plataforma REA, han sido desarrolladas bajo el enfoque de aprendizaje colaborativo y trabajo colaborativo apoyado en la computadora; el primero está relacionado con el ámbito educativo, el segundo, a ambientes de trabajo profesional que buscan el aprendizaje de técnicas y procedimientos específicos. Ambos están basados en la idea de que los sistemas electrónicos pueden facilitar los procesos y la dinámica de aprendizaje, en formas 
que no son realizables por medios de comunicación presenciales.

Por lo descrito anteriormente, se integran las bases conceptuales del conocimiento distribuido señaladas por Hollan \& Kirsh, 2000; Gibbons, Nelson \& Robert, 1998, dichos autores, proponen aplicar nuevos paradigmas de enseñanza que rompan con los límites impuestos por un enfoque tradicional de obtención de la información y tomar como base de posibilidades que ofrecen los medios electrónicos de comunicación e información. Se consideran como elemento fundamental los desarrollos tecnológicos, como Sistemas de Apoyo Electrónico al Rendimiento (Electronic Performance Support Systems, EPSS por sus siglas en inglés) o como los sistemas de disposición de recursos, pensados como objetos de aprendizaje, para apoyar una gran variedad de necesidades de educación y adiestramiento. Dichos recursos tienen la capacidad de potenciar el rendimiento y el aprendizaje, (texto, vídeo, pictogramas, gráficas, etc.), están organizados en conjuntos de datos, que llevan un mensaje relacionado con un tópico o tarea específicos. Estos sistemas ofrecen situaciones o ambientes flexibles de desarrollo o de creación de productos de enseńanza, en los que el significado de un recurso digital se define continuamente, situándolo en diferentes contextos, para satisfacer las necesidades del individuo que está en proceso de construcción de conocimiento.

\section{Diseño de la Arquitectura de la Plataforma REA para la Formación de Capital Humano en pymes}

Por lo que en este artículo se presenta la primera etapa que comprende la fase diagnóstica de esta tercera versión de la plataforma REA, la cual se ha ido migrando de dos versiones anteriores, iniciándose el proyecto de investigación en el 2015, en el Instituto Politécnico Nacional de México.

Fase diagnóstica, en la cual se hizo un levantamiento de información a 97 empresarios para unificar criterios y estándares, datos que sirvieron para elaborar el diseño y el desarrollo tecnológico REA, que se encuentra en prueba. Los hallazgos encontrados y que permitieron implementar la plataforma REA versión 3, se describen en la sección de resultados.
Fase de diseño y desarrollo de la plataforma, fueron una diversidad de herramientas que se integraron en el diseño, para que este recurso sea dinámico y acorde a las tendencias, como se puede visualizar en la secuencia de figuras tomadas de la plataforma. El menú se construyó para que los usuarios desarrollaran contenidos y posteriormente se muestren en un buscador por categorías, tal como lo muestra la figura 1 . El diseño de esta nueva plataforma REA 2018-2019, ha integrado como propuesta para su diseńo, herramientas digitales para cursos MOOC en una de sus secciones.

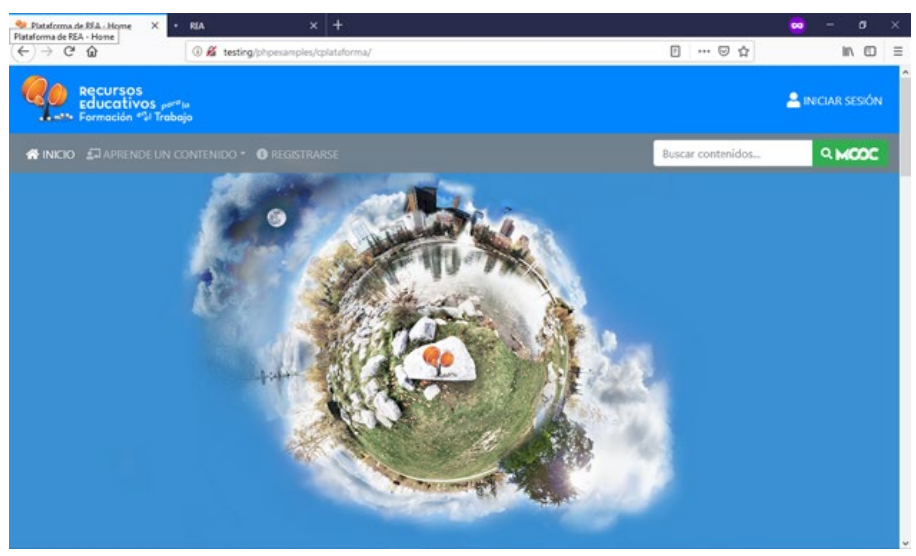

Figura 1

Plataforma REA 2018-2019

Fuente: Elaboración Propia (2018)

Un aspecto importante de esta tercera versión de la plataforma, es que alberga cursos MOOC, cuya característica es que son cursos masivos, el usuario es quien se registra directamente sin necesidad de depender de algún administrador o soporte técnico de alguna plataforma educativa. El registro en plataforma se muestra en la figura 2 .

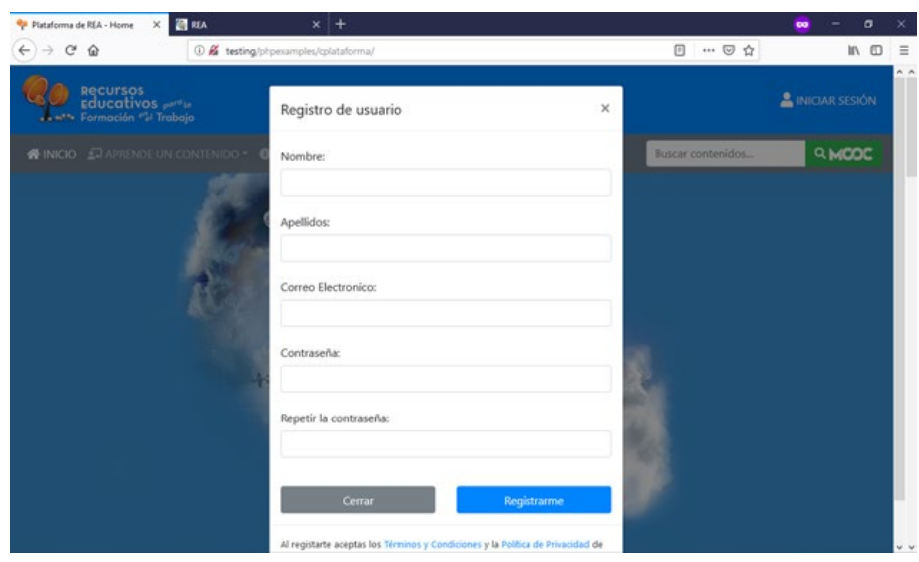

Figura 2

Registro en plataforma REA para cursos masivos MOOC

Fuente: Elaboración Propia (2019) 
Otra de las ventajas de esta tercera versión de la plataforma REA fue diseñada para que el usuario desarrolle de forma autónoma, y que el mismo sea el que haga un auto-monitoreo en el cumplimiento del objetivo planteado para cada tema.

\section{La Formación de capital humano}

En México y en el mundo se está desarrollando la cuarta revolución industrial, que se manifiesta en una gran complejidad e interconexión entre múltiples sectores, los cuales deben trabajar conjuntamente para entender mejor las tendencias emergentes (Schwab, 2016, p. 14). En este contexto, radica la importancia de la creatividad y la capacidad del capital humano para adaptarse y modificar los modelos de negocios y crear nuevos servicios en sus organizaciones para lograr un rendimiento y permanencia en esta nueva economía.

Vivimos en una economía basada en el conocimiento que surgió a partir de la tercera revolución industrial, donde el capital humano es la fuente de la riqueza que tienen las organizaciones. Siendo este capital humano al que debemos dedicar los esfuerzos de formación que garanticen la capacidad de creatividad e innovación continua, para emprender nuevos servicios y nuevos modelos de negocios. (Martínez, 2017, p. 222).

A partir de lo anterior, diversos estudios han permitido conocer la situación por la que atraviesan las pymes en México; Cueto (2013) plantea que entre los aspectos que más se enfrentan son, la preocupante tasa de mortalidad en este sector y el ciclo de vida que no va más allá de cinco años y solo el diez por ciento logran permanecer, es decir, solamente 2 de cada 100 empresas creadas logran mantenerse activas en el mercado. Es en este sentido que, la consolidación y madurez de una empresa sólo se logra a través de establecer estrategias que permitan desarrollar factores internos estratégicos como la administración del conocimiento, la innovación tecnológica en sus procesos y sus productos, el desarrollo del liderazgo en capital humano, la generación de estrategias de calidad y generar valor para ser más competitivas. Esto se fundamenta con datos de México Bursátil, 2013, en el que se muestra que el promedio de vida de las empresas ha aumentado a 7.7 años en prome- dio nacional (C.E. INEGI, 2015), esto se debe a las estrategias que se han seguido para consolidarlas en los mercados globales, sin embargo, aún las micro-empresas siguen siendo el mayor porcentaje de las Mipymes, es decir, las micro-empresas no logran convertirse en pequeñas empresas y posteriormente a medianas organizaciones, debido a los factores antes mencionados.

Padilla, Quintero \& Sales (2015) plantean que la formación del capital humano es un reto ineludible para la innovación empresarial, debido que los constantes cambios y la rapidez en que se modifican los negocios dejan fuera del plano la capacitación, existe una necesidad de formación del capital humano y existe una oportunidad de crear nuevos esquemas que fortalezcan las capacidades de las empresas en diversos ámbitos relacionados a los negocios.

Por otra parte, si tomamos como base los resultados que arroja el quinto informe de las tendencias globales de capital humano publicado por Deloitte, se habla de un panorama enteramente digital en donde se vive la era de la disrupción, esto significa que las organizaciones se ven en la necesidad de reinventar estrategias para mejorar y optimizar los procesos que impacten en mejores productos y servicios. Se trata de una oportunidad para crear plataformas, procesos y herramientas que continúen evolucionando y manteniendo su valor con el tiempo. Deloitte (2017). En dicho estudio se demuestra la brecha tan amplia que existe entre la productividad y el acelerado cambio tecnológico, esto significa que las empresas que no empleen la tecnología en la optimización de sus procesos, están destinadas a desaparecer del mercado.

Por lo anteriormente expuesto, es importante proveer a las pymes de esquemas de formación de capital humano, en el que no lo consideren un gasto, sino una inversión, por esta razón es importante llevar al ámbito del trabajo la actualización del conocimiento, lo cual repercutirá en el aumento de la productividad y por ende el crecimiento económico, (Kido, A. \& Kido, T, 2015; Hirsch, Almaraz $\&$ Ríos, 2015). Una de las estrategias que permite al capital humano a continuar con su formación sin interferir con otras actividades ya programadas y gestionar su tiempo y estilo de aprendizaje en 
los trabajadores, es a través de recursos educativos abiertos implementados en plataformas tecnológicas amigables y que atiendan las necesidades inmediatas para resolver problemas que en el día a día se enfrentan. Esto implica, facilitar la formación atendiendo a necesidades de espacio y tiempo del usuario, es decir, que las personas se sientan con la libertad de llevar su formación, sin necesidad de trasladarse a un espacio específico y con horarios rígidos que no le ayuden a vivir la formación como un proceso de aprendizaje que le beneficie en su desarrollo personal y laboral.

Todos los nuevos desarrollos y tecnologías tienen una característica clave en común: aprovechan el poder de penetración que tienen la digitalización y las tecnologías de la información... La revolución digital está creando enfoques radicalmente nuevos que revolucionan la forma en que los individuos e instituciones participan y colaboran entre sí. (Schwab, 2016. p.21)

\section{Materiales y Métodos}

\section{Participantes}

Los sujetos que participaron en la investigación fueron 97 empresarios, quienes lo hicieron de manera voluntaria, pertenecientes a los diversos sectores de servicios, de la Delegación Tlalpan de la Ciudad de México que constituyen el universo de la población. El tipo de muestreo fue no probabilístico y la selección de la muestra fue por conveniencia, atendiendo a la disponibilidad de los sujetos e interesados en participar en el estudio y su deseo de ser formado a través del uso de los Recursos Educativos Abiertos de la Plataforma.

\section{Instrumento}

Se utilizó el método cuantitativo de recopilación de información para la etapa de diagnóstico a sujetos de estudio, quienes tuvieron un acercamiento al uso de los Recursos Educativos Abiertos de la Plataforma, a través de un cuestionario de respuestas de opción múltiple y de escala Likert, mediante el cual se obtuvo información sobre aspectos sociodemográficos, aspectos de su formación en cuestiones tecnológicas, así como las preferencias sobre el uso de tecnologías para su formación en el trabajo, dicho cuestionario fue validado por la técnica de juicio de expertos en el que se realizó un análisis profundo del contenido de cada uno de los ítems, teniendo una validez en promedio del $90 \%$ por los cinco expertos, con el levantamiento de pequeñas observaciones a los ítems .

Los resultados obtenidos en este estudio sirvieron como base para desarrollar las etapas de diseño y aplicación de la Plataforma REA.

\section{Tipo y diseño}

Se realizó una investigación de campo de tipo cualitativa, exploratoria-descriptiva presentada como diagnóstico, y con la cual se plantea el desarrollo de la Plataforma en Recursos Educativos Abiertos para ser utilizada en la formación de capital humano en pymes del ramo de empresas de servicios de la Delegación Tlalpan de la Ciudad de México, para aumentar su permanencia en el mercado y mejorar sus productos y servicios.

\section{Procedimiento}

A continuación, se describe las dos fases que integra los resultados en este estudio, quedando para estudios subsecuentes la aplicación y validación de la tercera versión en el desarrollo de la Plataforma Tecnológica en cuanto a la Formación de Capital Humano a pyme a través de contenidos digitales, entendidos como Recursos Educativos Abiertos (REA) para la Formación en el Trabajo, son:

\section{Fase Diagnóstico}

- Estudio Diagnóstico de los usuarios de la plataforma. - Participaron 97 sujetos de estudio, identificados como empresarios/directivos de las pymes de la Delegación Tlalpan de la Ciudad de México, lo cual hace una diferenciación con la publicación de estudios de versiones anteriores por el tipo de muestreo utilizado. En este estudio se presenta esta primera fase diagnóstica.

- Fase diseño de la plataforma para integrar MOOC 
1. En el proyecto de la nueva plataforma REA 2018-2019 se diseña en una arquitectura más estable para el desarrollo de contenidos, con la intención de albergar cursos MOOC que permita alojar a más usuarios en corto tiempo.

2. La versión 3 de la plataforma se creó para atender la formación de capital humano en las pymes y su formato se realizó de la misma manera en que se concibió la plataforma REA en sus versiones 1 y 2 .

3. La versión 3 también contiene categorías según la departamentalización clásica de la empresa.

4. Cada Contenido Digital mantiene en su estructura: título del contenido, objetivo de aprendizaje, contenido digital, actividad y evaluación del aprendizaje, al igual que la versión 1 y2.

5. El desarrollo de la Arquitectura de la plataforma tecnológica, se modifica en el diseño de la interfaz, con una estructura de base de datos que permite almacenar, registrar, manipular y editar contenidos digitales de manera más amigable y de fácil acceso, que las versiones anteriores.

6. El desarrollo de los administradores de contenido, conservan la categoría y campos acorde a la estructura de metadatos igual que la versión 1 y 2 de la plataforma, esto con el propósito de controlar y dar seguimiento a la subida de contenidos digitales, empleando estándares SCORM, para organizar y monitorear las actividades.

\section{Consentimiento informado o Confidencialidad}

El consentimiento para la participación de los sujetos participantes, se obtuvo a través de pláticas informativas, asegurando la confidencialidad de su participación al mencionar que la información obtenida es utilizada únicamente para fines de investigación y académicos.

En relación al uso de la Plataforma REA como es de desarrollo propio del equipo de investigación, no hubo necesidad de pedir consentimiento en su uso para fines de investigación.

\section{Resultados}

Fase diagnóstica

A continuación, se muestran los resultados obtenidos al aplicar el cuestionario a los posibles usuarios de la Plataforma REA, en cuanto a aspectos sociodemográficos, su formación en cuestiones tecnológicas, necesidades de capacitación, así como las preferencias sobre el uso de tecnologías para su formación en el trabajo.

\section{Datos Generales}

La evaluación de la fase diagnóstica se realizó con los empresarios del sector servicios, que estaban interesados en formar a su capital humano a través de la Plataforma REA para crear nuevos servicios. En la figura 3 se muestra en porcentajes el género de los empresarios participantes en el estudio.

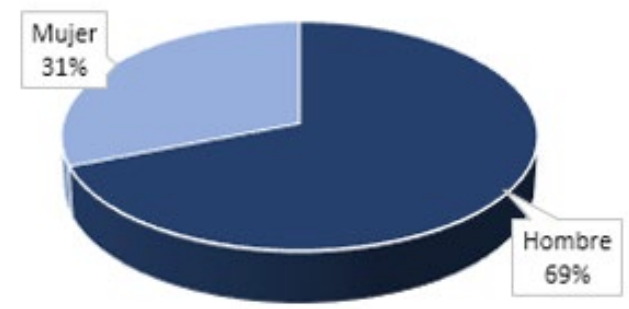

Figura 3

Género de la muestra.

Fuente: Elaboración Propia (2018)

La participación del género masculino (69\%) en la dirección de las organizaciones, sigue siendo mayor, sin embargo, ha existido un aumento en la participación de la mujer (31\%), a diferencia del anterior estudio realizado en el proyecto SIP 20150905, (Martínez \& Chávez, 2016).

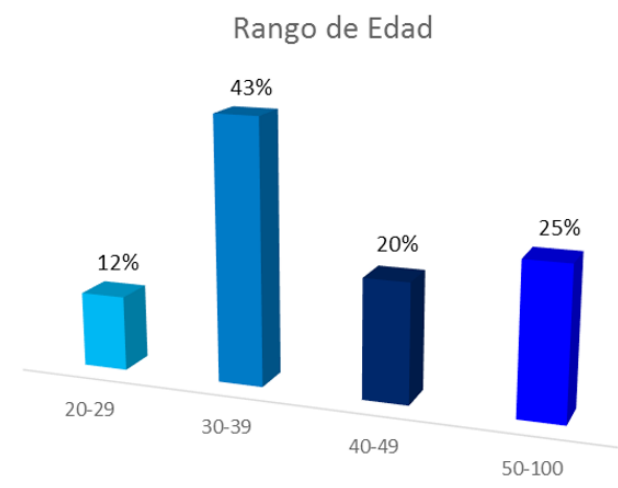

Figura 4

Rango de edades de la muestra.

Fuente: Elaboración Propia (2018) 
Otro factor importante a considerar es la edad de los empresarios, debido a que atraviesan por una etapa de transición, debido a que tienen en promedio entre 30 a 40 años de edad (43\%), como se observa en la figura 4 .

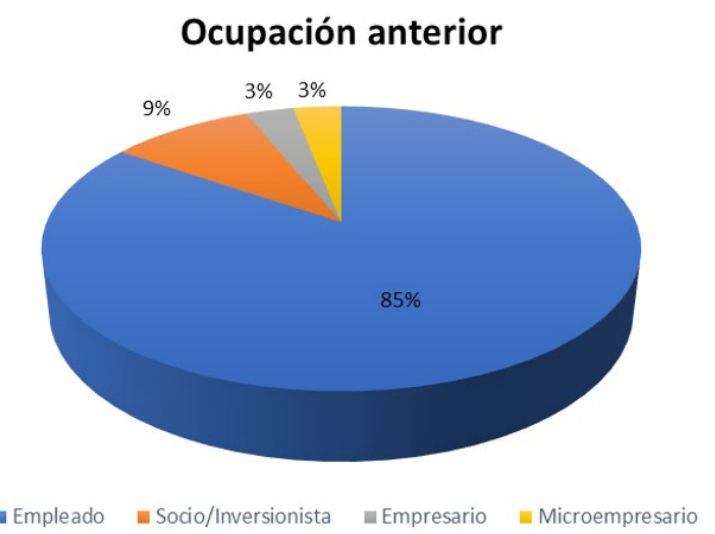

\section{Figura 5}

Ocupación anterior.

Fuente: Elaboración Propia (2018)

Como se describe en la figura 5 , el $85 \%$ de los participantes menciona que fueron empleados en alguna organización antes de ser empresarios y como dato interesante, se encontró que la sucesión de empresas representa solo el 3\%, por otra parte, el crecimiento de microempresarios a empresarios es apenas del 3.\%, lo que nos indica que son pocas las empresas que sobreviven al crecimiento y otras simplemente no pueden crecer, lo que probablemente se relaciona con la falta de capacitación y actualización de los empresarios o quizás a otros factores internos y externos.

En cuanto al uso de las tecnologías y medios digitales para la búsqueda de información, se brindó la alternativa de marcar más de una opción de acuerdo a la prioridad del dispositivo, los datos obtenidos de la investigación muestran que los empresarios emplean con mayor frecuencia en un 96\% el teléfono móvil para buscar información, quizás se deba a que este lo tienen disponible en todo momento. (Ver figura 6).

Es importante destacar que los empresarios, tienen también una preferencia hacia la laptop antes que a una Tablet, probablemente debido a que en una laptop, pueden mantener organizados documentos necesarios para sus actividades operativas
Uso de dispositivos de búsqueda de información

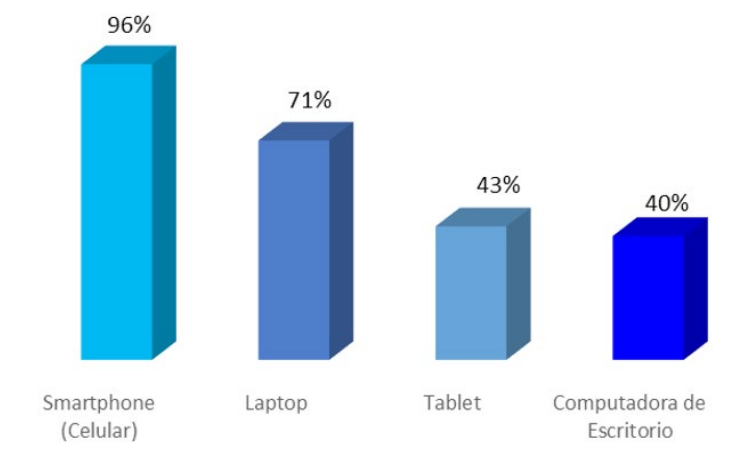

Figura 6

Dispositivos empleados para búsqueda de información. Fuente: Elaboración Propia (2018)

y pueden fácilmente editarlas en una pantalla más amplia, pueden agendar sus citas, revisar expedientes y estas pueden ser transferidas a sus empleados, por otra parte se encuentran los precios de adquisición y la flexibilidad que ofrecen de ser transportadas entre salas de juntas $u$ oficinas de clientes o proveedores, ofrecen una interoperabilidad mayor que aquellos dispositivos que requieren internet para manejar archivos, además su potencia de procesamiento de datos es mayor.

En la Figura 7 se muestra los temas que generan mayor interés en los empresarios, por lo que se tomaron en cuenta para integrarlos en la formación de capital humano en el trabajo a través de la plataforma REA, entre los aspectos más relevantes se observa que el $69 \%$ indicó que le gustaría conocer más sobre el análisis de proyectos de inversión, el $58 \%$ de ellos buscan temas relacionados con la administración financiera, el 53\% propone temas de sustentabilidad y el $44 \%$ se interesa por aspectos relacionados con la toma de decisiones.

Temas prioritarios en Internet para capacitación

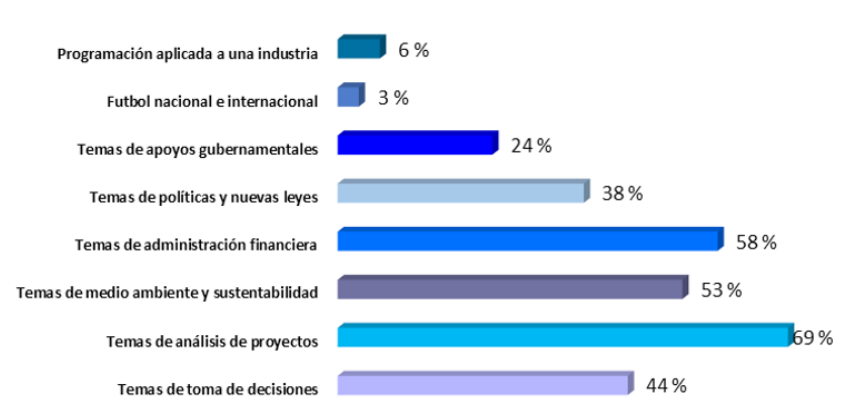

Figura 7

Temas prioridad en internet

Fuente: Elaboración Propia (2018) 
Es evidente que todos los temas seleccionados son fundamentales para las organizaciones, ya que contribuyen a determinar las áreas en que es recomendable la inversión para el desarrollo y formación en talento humano, con la finalidad de permanecer en el mercado y, de ser factible, emprender estrategias de crecimiento.

Respecto a la preferencia del uso de dispositivos para recibir cursos de capacitación, en la Figura 8 se observa que los empresarios prefieren los cursos presenciales (48\%), sin embargo, a diferencia de haber indicado que utilizan más el teléfono móvil para buscar información, en el caso de recibir capacitación el 34\% muestran interés por realizarlo a través de internet mediante el uso de la computadora, lo que indica que es posible generar contenidos digitales que serán aceptados por los empresarios.

\section{Medio por el que recibiría el curso}

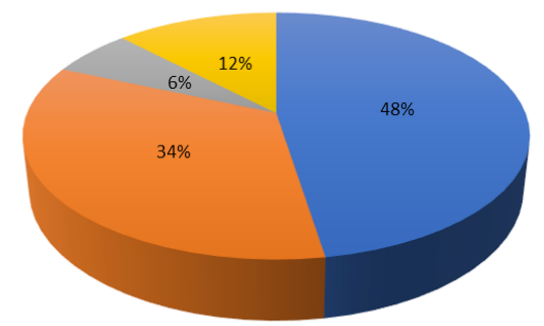

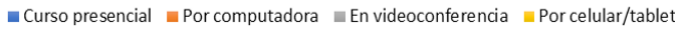

\section{Figura 8}

Medios para recibir la capacitación.

Fuente: Elaboración Propia (2018)

\section{Discusión y Conclusiones}

Dentro de las problemáticas que presentan las pymes para la formación de su capital humano, destacamos que no existe en ellas una adecuada gestión del conocimiento para su crecimiento constante, es decir además de no tener la capacidad de adquirir capacitación y formación constante por medio de las instituciones educativas, no existen en ellas herramientas que gestionen su información para lograr la adecuada formación del capital humano, generalmente este conocimiento se transmite bajo el esquema de adiestramiento, esto lo confirma Tavera \& Salinas(2011) quienes mencionan que las pymes presentan una serie de problemas relacionados con el desconocimiento en negocios, como la planeación estratégica, el desarrollo de una cultura empresarial, la innovación tecnológica, que limitan la competitividad y la productividad de las mismas, destacando que no existe en ellas un plan de negocios desarrollado.

La formación del capital humano a través de recursos educativos abiertos en las organizaciones, ofrece a los usuarios, elementos muy valiosos en su formación, si se considera su disponibilidad, rapidez de acceso y actualización, así como su bajo costo y adaptabilidad. Lo cual contribuirá a la formación de y fortalecimiento del capital humano de alto nivel, (Romero, López, \& Martínez, 2018).

Los hallazgos encontrados en la presente investigación, cimentan e inciden en la importancia de hacer una reingeniería en la formación del capital humano, en la que las tecnologías formen parte de los recursos que tienen a su alcance y que deben apropiarse de ellas, y que sean integradas como una estrategia en el ámbito organizacional y empresarial, ya que el tema de las tecnologías en el ámbito de la industria para los empresarios todavía en este siglo XXI no está siendo una prioridad según los empresarios encuestados al indicar que solo el $6 \%$ de ellos están de acuerdo en ser necesario la programación aplicada en el sector industrial, lo cual se muestra en la problemática que aún existe en cuanto a la apropiación de las tecnologías en diversos ámbitos empresariales, Padilla, Quintero \& Sales (2015), al respecto plantean que la formación del capital humano es un reto ineludible para la innovación empresarial, debido que los constantes cambios y la rapidez en que se modifican los negocios dejan fuera del plano la formación, existe una necesidad de formación del capital humano y existe una oportunidad de crear nuevos esquemas que fortalezcan las capacidades de las empresas en diversos ámbitos relacionados a los negocios. Además, es una de las capacidades de las organizaciones para lograr una ventaja competitiva y que tiene una relación directa con el conocimiento y habilidades de capital humano, (Páez, 2012).

El 34\% muestran interés por realizarlo a través 
de internet mediante el uso de la computadora, lo que indica que es posible generar contenidos digitales que serán aceptados por los empresarios.

Los resultados mostrados en cuanto a seguir cursos a través de internet mediante el uso de la computadora, (34\%) es un referente para poder realizar plataformas idóneas para la formación de capital humano con contenidos digitales que serán aceptados por los empresarios, por la que en estas se utilicen diversas herramientas o medios digitales tales como el video, gráficos, sonido, presentaciones interactivas, infografías, nubes de tags, etc., que apoyen el diseño y desarrollo de contenidos digitales, entendidos éstos como recursos educativos abiertos, para crear ambientes de aprendizaje virtual más dinámicos, esto contribuye a favorecer en el adulto un aprendizaje más situado a su realidad laboral, permitiendo de manera paralela, aplicarlo a la solución de problemas a los que se enfrenta en su ámbito laboral en el desempeño de sus funciones.

El diagnóstico realizado ha permitido, el diseño y desarrollo de la plataforma REA, en la que se trabaja actualmente con herramientas open source en ambientes virtuales de aprendizaje, esto permite a las empresas, la constante formación en el trabajo del capital humano de manera accesible, a bajo costo, acorde al contexto y a las necesidades del grupo destinatario. Tal es el caso de las plataformas de recursos educativos abiertos que se basan en recursos disponibles para todo el público, generalmente son materiales pedagógicos libres, gratuitos $y$ accesibles en la red, bajo licencias que permiten su reutilización, de acuerdo al consorcio OCW (Open Course Ware Consortium) es un conjunto especifico y estructurado de alta calidad que incluyen generalmente materiales para la planificación, evaluación y estructura de los contenidos.

En este mismo sentido, existen experiencias en universidades públicas en México como la Universidad Nacional Autónoma de México, a través de la Coordinación Universidad Abierta y Educación a Distancia (CUAED) y el IPN (Instituto Politécnico Nacional), que han desarrollado plataformas digitales para lograr satisfacer la necesidad de formación en el ámbito de las organizaciones, sin embargo, la demanda aumenta radicalmente, tanto en sectores productivos como de servicios e incluso el sector privado ha incursionado en el negocio de la formación, generando plataformas con programas y cursos atractivos a un precio considerablemente económico. Butcher (2015) menciona que las oportunidades de aprendizaje se deben proporcionar a todos los individuos a lo largo de la vida y proporcionarles los medios y herramientas tecnológicas para desarrollar competencias digitales que les permita estar integrados en la sociedad de la información y el conocimiento distribuido.

Se considera importante mencionar que para consolidar el diseño y desarrollo de la Plataforma REA en su versión 3 en la integración de cursos MOOC, se pretende, en futuros estudios, integrar herramientas digitales que desarrollen las habilidades del pensamiento estratégico en los empresarios, para favorecer el trabajo en equipo, brindar un conocimiento colectivo enfocado en la solución de problemas, favorecer las redes de negocios para propiciar soluciones a clientes con expertos en los temas, esto con la finalidad de brindar certeza entre los integrantes de las organizaciones y certidumbre en la formación del capital humano en la organización.

Para futuras investigaciones sobre el diseño y desarrollo de plataformas tecnológicas donde se utilicen los Recursos Educativos Abiertos, se debe hacer una batería de instrumentos fiables y confiables para recopilar información de las necesidades que tienen y requieren los usuarios de este tipo de plataforma para su mejora en su formación como capital humano.

\section{Agradecimientos}

Al proyecto SIP 20161051 Instituto Politécnico Nacional: Aplicación de la Metodología para Validar Plataforma Tecnológica Desarrollo de Contenidos Digitales para la Formación en el Trabajo a Empresarios pymes de la Delegación Xochimilco.

\section{REFERENCIAS BIBLIOGRÁFICAS}

Butcher, N. (2015) Guía Básica de Recursos Educativos Abiertos (REA). Place de Fontenoy, 75352 París 07 SP, 
Francia: Organización de las Naciones Unidas para la Educación, la Ciencia y la Cultura.

Cámara de Comercio (2017) Tecnología aplicada al capital humano se enfoca en la evaluación de desempeño. Informe especial Cámara de Comercio de Lima, Perú. La revista de la Cámara de Comercio, No.791, 13-15. Recuperado de https:/www.camaralima.org.pe/repositorioaps/0/0/par/edicion791/edicion\%20791.pdf

Censos Económicos, C.E. INEGI. (2015). Micro, pequeña, mediana y gran empresa estratificación de los establecimientos. INEGI. Aguascalientes, México: Instituto Nacional de Estadística G.

Cueto, A. (2013). Capability Maturity Model (CMM) y la Gerencia. Col. Laboratori de Mitjans Interactius / Publicacions i Edicions de la Universitat de Barcelona. ombia: I Jornadde Gerencia de Proyectos en TI - ACIS 2003.

De Marco, F. (2013) El capital humano como fuente de Innovación en una organización académica. GTEC. Centro -Este. Programa de apoyo al gerenciamiento de las innovaciones tecnológicas.

Deloitte. (2017). 5to Reporte y Encuesta anual de tendencias globales de Capital Humano. México City: Deloitte.

Diaz, H. (2017) Tecnologías de la información y comunicación y crecimiento económico. Economía Informa, 405.

Gálvez, E., Riascos, S. \& Contreras, F. (2014) Influencia de las tecnologías de la información y comunicación en el rendimiento de las micro, peque-as y medianas empresas colombianas. Estudios Gerenciales, 30, 355-364. https://doi. org/10.1016/j.estger.2014.06.006

Gibbons, A., Nelson, J. \& Robert, R. (1998) Learning object design and sequencing theory in David Wiley (Ed.), Designing Instruction with Learning Objects, June.2000

Guzmán, J. \& Motz, R. (2013) Considerando equivalencias pedagógicas para la reutilización de recursos educativos abiertos. Revista Iberoamericana de Educación en Tecnología y Tecnología en Educación, 11, 8-17.

Hill, J. \& Hannafin, M. J. (2000). Teaching and learning in digital environments: The resurgence of resource-based learning. Submitted for publication.

Hirsch, J., Almaraz, I. \& Ríos, M. (2015) La preparación de las empresas manufactureras del Estado de Querétaro, México en el área de las tecnologías de información y comunicación. Suma de Negocios, 6, 166-177. https://doi. org/10.1016/j.sumneg.2015.08.012

Hollann, J., E. \& Kirsh, D. (2000) Distributed Cognition: Toward a new Fundation for Human-Computer Interaction Research. ACM Transaction Computer-Human Interaction 7(2),174-196 https://doi.org/10.1145/353485.353487

Kido, A. \& Kido, T. (2015) Modelos teóricos del capital humano y señalización: un estudio para México. Contaduría y Administración, 60, 723-734. https://doi.org/10.1016/j. cya.2014.06.001

Martínez, F. (2017). Administración Estratégica Inteligente. El pensamiento Estratégico en la 4ta. Revolución Industrial y su economía de Creatividad e Innovación. Ciudad de México: Instituto Mexicano de Innovación y Estrategia, A.C. https://doi.org/10.25100/cdea.v32i56.4284

Martínez, M. \& Chávez, D, (2016) Desarrollo de plataforma tecnológica para la formación de capital humano en PyMES de la ciudad de México. XXI Congreso internacional de contaduría administración e informática. Recuperado de http:// congreso.investiga.fca.unam.mx/docs/xxi/docs/3.03.pdf

Mejía, A. (2017) Personal calificado en la industria de las TICs en México: El caso de las MIPYMES. Gestión de la Innovación para la Competitividad. Altec 2017, XVII Congreso Latino Iberoamericano de Gestión Tecnológica, México.

México Bursátil. (26 de Septiembre de 2013). Los principales problemas de las PyMES. Recuperado de http://www. mexicobursatil.com/los-principales-problemas-de-las-pymes/

Ortega, J. A., Hernández, A. M., Martínez, M. D., Rendón, L. M., Fuentes, J. A., Pérez, A. \& Ortega, A. (2013). La educación a distancia en entornos virtuales hoy. GEU México.

Padilla, S., Quintero, L., \& Sales, J. (2015). Dinámicas grupales en la capacitación empresarial por competencias. Las micro, pequeñas y medianas empresas en el desarrollo económico, cultural y tecnologico de México, 63-74.

Páez, I. (2012) Capital humano, redes externas e innovación en la industria colombiana. Estudios Gerenciales, 28, edición especial, 81-107. https://doi.org/10.18046/j.estger.2012.1480

Reyes, B. Juárez, C., Martínez, M. \& Sánchez, J. (2017) Modelo de propiedad intelectual para recursos educativos abiertos. Campus Virtuales, 6 (2), 107-112. Recuperado de http:// www.uajournals.com/campusvirtuales/journal/11/9.pdf

Román, M. (2013). Crecimiento en un contexto de escasez de capital humano en TIC. Tercera Conferencia en Desarrollo Capital Humano. Universidad Tecnológica de Chile. INACAP.

Romero, J., López, V. \& Martínez, M. (2018) El capital humano en México en el marco de la economía basada en el conocimiento: Una revisión a los indicadores de ciencia y tecnología. Economía coyuntural. Revista de temas de coyuntura y perspectivas, 3 (1) 1-36.

Salomon, E. G. (2005) Distributed Cognition: Psychological and Educational Considerations, Cambridge University Press, Cambridge, United Kingdom.

Schwab, K. (2016). La cuarta revolución industrial. México: Penguin Random House.

Somerville, H. (2018). U.S.News, Technology News. Recuperado de Udacity, With Eye to Eventual IPO, Says Revenue More Than Doubled in 2017: https:/www.usnews.com/ news/technology/articles/2018-02-27/udacity-with-eye-toeventual-ipo-says-revenue-more-than-doubled-in-2017

Tavera, M. E., \& Salinas, E. (2011). Las MIPyME en México. Crecimiento, financiamiento y tecnología. México D.F.: Instituto Politécnico Nacional. 